$$
\begin{aligned}
E & =\text { total energy, } \\
2 \pi \hbar & =\text { Planck's constant, } \\
a & =\text { radius of the well. }
\end{aligned}
$$

Using this table, the first few roots have been obtained graphically and are recorded in Table 1 to three significant digits. For most practical purposes, these values should be satisfactory. If necessary, they can be improved by use of Newton's method.

Polytechnic Institute of Brooklyn

Brooklyn, New York

1. M. Onoe, Tables of Modified Quotients of Bessel Functions of the First Kind for Real and Imaginary Arguments, Columbia University Press, New York, 1958.

\title{
A Note on Factors of $n^{4}+1$
}

\section{By A. Gloden}

The factorizations enumerated in this note form a sequel to my published factor table [1] of integers $n^{4}+1$. They have been obtained by means of my table of solutions of the congruence $x^{4}+1 \equiv 0(\bmod p)$ for primes lying between $8 \cdot 10^{5}$ and $10^{6}[2]$.

The following numbers are primes:

$$
\begin{aligned}
& n^{4}+1 \text { for } n=912,914,928,930,936,952,962,966,986,992,996 . \\
& \frac{1}{2}\left(n^{4}+1\right) \text { for } n=1071,1087,1101,1119,1123,1125,1135,1163,1173,1183 . \\
& \frac{1}{17}\left(n^{4}+1\right) \text { for } n=1562,1726,1732,1834 \text {. } \\
& \frac{1}{41}\left(n^{4}+1\right) \text { for } n=1818,1848,1982,2006,2012,2064,2088,2094,2228,2340 \text {, } \\
& 2364 . \\
& \frac{1}{7 \overline{3}}\left(n^{4}+1\right) \text { for } n=2346 \text {. } \\
& \frac{1}{89}\left(n^{4}+1\right) \text { for } n=2262,2302,2544,2682 . \\
& \frac{1}{113}\left(n^{4}+1\right) \text { for } n=2468 \text {. } \\
& \frac{1}{187}\left(n^{4}+1\right) \text { for } n=2476 \text {. } \\
& \frac{1}{283}\left(n^{4}+1\right) \text { for } n=2808 \text {. } \\
& \frac{1}{2 \cdot 17}\left(n^{4}+1\right) \cdot \text { for } n=1709,1715,1759,1787,1827,1845,1855,1879,1895,1963 \text {, } \\
& 2015,2021,2031,2093,2185,2229,2259,2287,2303 \text {, } \\
& \text { 2327, } 2331 . \\
& \frac{1}{2 \cdot 41}\left(n^{4}+1\right) \text { for } n=2211,2299,2651,2761,2791,2815 \text {. } \\
& \frac{1}{2 \cdot 7.3}\left(n^{4}+1\right) \text { for } n=2533,2577,2691,2723,2857 \text {. }
\end{aligned}
$$

Received June 8, 1959. 
$\frac{1}{2 \cdot 89}\left(n^{4}+1\right)$ for $n=2747,2771,2885$.

$\frac{1}{2 \cdot 97}\left(n^{4}+1\right)$ for $n=2669,2683,2749$.

New factorizations are as follows:

$$
\begin{aligned}
938^{4}+1 & =809273 \cdot 956569 \\
1060^{4}+1 & =847577 \cdot 1489513 \\
1348^{4}+1 & =940169 \cdot 3511993 \\
1512^{4}+1 & =926617 \cdot 5640361 \\
1874^{4}+1 & =914561 \cdot 13485457 \\
2100^{4}+1 & =17 \cdot 873553 \cdot 1309601 \\
2838^{4}+1 & =868841 \cdot 74663657 \\
2908^{4}+1 & =41 \cdot 940369 \cdot 1854793 \\
\frac{1}{2}\left(1155^{4}+1\right) & =830233 \cdot 1071761 \\
\frac{1}{2}\left(1191^{4}+1\right) & =935353 \cdot 1075577 \\
\frac{1}{2}\left(1509^{4}+1\right) & =872369 \cdot 2971849 \\
\frac{1}{2}\left(2635^{4}+1\right) & =857569 \cdot 28107577 \\
\frac{1}{2}\left(2765^{4}+1\right) & =908353 \cdot 32173321 \\
\frac{1}{2}\left(2977^{4}+1\right) & =17 \cdot 809041 \cdot 2855393
\end{aligned}
$$

The following factorization was omitted from my original table [1]:

$$
\frac{1}{2}\left(2055^{4}+1\right)=17 \cdot 572233 \cdot 916633 .
$$

The least integers still incompletely factored correspond to $n=1038$ and 1229 , for even and odd values of $n$, respectively.

11 rue Jean Jaurès

Luxembourg

1. A. Gloden, "Table de factorisation des nombres $n^{4}+1$ dans l'intervalle $1000<n<$ 3000," Institut Grand-Ducal de Luxembourg, Archives, Tome XVI, Luxembourg, 1946, p. 7188.

2. A. Gloden, Table des Solutions de la Congruence $x^{4}+1 \equiv 0(\bmod p)$ pour $800,000<p$ $<1,000,000$, published by the author, rue Jean Jaures, 11, Luxembourg, 1959.

\section{A Note on the Solution of Quartic Equations}

\section{By Herbert E. Salzer}

For any quartic equation with real coefficients,

$$
X^{4}+A X^{3}+B X^{2}+C X+D=0,
$$

the following condensation of the customary algebraic solution is recommended as quickest and easiest for the computer to follow (no mental effort required). It works in every exceptional case.

Received December 22, 1959. 